\title{
О ВЛИЯНИИ ВОДОРОДА И АЛКИЛЬНОГО ЗАМЕСТИТЕЛЯ НА ПОТЕНЦИАЛЫ ИОНИЗАЦИИ
}

Изменение потенциалов ионизации (ПИ) молекул при введении алкильных групп обусловлено главным образом индукционным эффектом присоединяемых заместителей $\left[{ }^{1,2}\right]$. Согласно теории, развитой в работах $\left[{ }^{3-5}\right]$, индукционное влияние есть результат последовательной поляризации ковалентных связей. Оно подчиняется с довольно удовлетворительной точностью закону затухания и правилу аддитивности. Применимость этих двух закономерностей для оценки изменения ПИ под влиянием алкильных заместителей показана в $\left[{ }^{1,2,6}\right]$. Исходя из правила аддитивности и формулы затухания индукционного влияния $\left[{ }^{6}\right]$, в работе $\left[{ }^{7}\right]$ выведено уравнение для вычисления индукционных констант $\mu^{*}$ алкильных заместителей:

$$
\mu^{*}=-0,1\left(i_{1}+i_{2} z+i_{3} z^{2}+\ldots+i_{n} z^{n-1}\right),
$$

где 0,1 - коэффициент для приведения масштаба в соответствие с тафтовской $\sigma^{*}$ шкалой; $z-$ фактор затухания, т. е. постоянная, характеризующая затухание индукционного влияния на одной группе $-\mathrm{CH}_{2}-$; здесь величина $z=0,5$ взята из $\left[{ }^{7}\right] ; n-$ показатель положения углеродного атома по отношению к хромофорной группе $\mathrm{Y}$ согласно схеме:

$$
\text { 114ave }
$$

$i_{1}, i_{2}, \ldots, i_{n}$ - число углеродных атомов в разных положениях относительно Y.

В работе $\left[{ }^{8}\right]$ предложены новые шкалы констант алкильных заместителей $\mu_{\mathrm{R}}$ и $\mu^{\prime}$, определенные из фотоэлектронно-спектроскопических и фотоионизационных данных. Константы $\mu_{\mathrm{R}}$ и $\mu^{\prime}$ отражают вызванное заместителем изменение ПИ относительно метилзамещенного соединения. Масштаб шкалы определяется выбором стандартной серии. В $\left[{ }^{8}\right]$ в качестве стандарта были использованы алкилиодиды. С помощью этих констант можно вычислить ПИ алкильных соединений с точностью до 0,05 эв. Согласно $\left[{ }^{8}\right]$, исключением является водород, который не имеет постоянного значения $\mu^{\prime}$. 
Однако авторы ряда работ предполагают, что водородный и алкильный заместители аналогично влияют на ПИ. Например, в $\left[{ }^{2,9-11}\right]$ показано, что между индукционными константами $\sigma_{I}$ или $\sigma^{*}$ и ПИ существует (хотя не очень точная) линейная зависимость в случае этих заместителей.

В настоящей работе сопоставляются шкалы индукционных констант, приведенные различными авторами, а также изучается их применимость для корреляции ПИ соединений с алкильными и водородным заместителями. Особое внимание уделяется водородному заместителю.

\section{Шкалы индукционных констант алкильных заместителей. Значение $\mu^{*}$ для водорода}

Мерой индукционного влияния заместителя обычно служат константы $\sigma^{*}$ Тафта $\left[{ }^{5}\right]$. Кажется целесообразным привести масштабы шкал констант $\mu_{\mathrm{R}}$ и $\mu^{\prime}$ в соответствие с масштабом величин $\sigma^{*}$ Тафта, так как масштаб определяется произвольным выбором стандартной серии. Предполагая точную применимость закона затухания и правила аддитивности к оценке изменений ПИ, находим, что шкала $\mu^{*}$ отличается от шкал $\mu_{\mathrm{R}}$ или $\mu^{\prime}$ только масштабом и что можно ожидать соблюдения следующих зависимостей:

$$
\begin{aligned}
\mu^{*} & =\frac{I_{\mathrm{RY}}-I_{\mathrm{CH}_{3} \mathrm{Y}}}{10\left(I_{\mathrm{CH}_{3} \mathrm{Y}}-I_{\mathrm{C}_{2} \mathrm{H}_{5} \mathrm{Y}}\right)}, \\
\mu^{*} & =\frac{\mu_{\mathrm{R}}}{10\left[\mu_{\mathrm{C}_{2} \mathrm{H}_{5}}\right]},
\end{aligned}
$$

где $I_{\mathrm{RY}}, I_{\mathrm{CH}_{3} \mathrm{Y}}, I_{\mathrm{C}_{2} \mathrm{H}_{5} \mathrm{Y}}-$ ПИ соединений с данным алкильным, метильным и этильным заместителями соответственно; знаменатели определяют масштаб.

Как видно из табл. 1, вычисленные значения константы $\mu^{*}$ хорошо согласуются с результатами $\left[{ }^{8}\right]$. Явное отличие обнаруживается только для третичных алкилов $\mathrm{C}\left(\mathrm{CH}_{3}\right)_{3}$ и $\mathrm{C}\left(\mathrm{C}_{2} \mathrm{H}_{5}\right)\left(\mathrm{CH}_{3}\right)_{2}$, для которых правило аддитивности, видимо, соблюдается не точно.

Довольно грубое выравнивание масштаба по началу шкал оправдывается наибольшей точностью ПИ первых членов серии, так как для водородного, метильного и этильного заместителей ПИ определены намного точнее, чем для остальных случаев.

Следующие довольно точные корреляционные уравнения (4) и (5) также показывают, что основное различие трех сравниваемых шкал проявляется в масштабе:

$$
\begin{gathered}
\mu^{*}=0,586 \mu_{\mathrm{R}}+0,013, \\
r=0,997, \quad s=0,007 \text { единиц шкалы; } \\
\mu^{*}=0,582 \mu^{\prime}+0,012, \\
r=0,986, \quad s=0,012 \text { единиц шкалы, }
\end{gathered}
$$

где $r$ - коэффициент корреляции, $s-$ стандартное отклонение.

Значительно меньшую точность имеет зависимость величин $\mu^{*}$ от констант Тафта:

$$
\begin{gathered}
\mu^{*}=1,166 \sigma^{*}-0,005 \\
r=0,946, \quad s=0,035 \text { единиц шкалы. }
\end{gathered}
$$

Параметры уравнений (4)-(6) определены методом наименьших квадратов. 
Сопоставление значений $\mu_{\mathrm{R}}, \mu^{\prime}, \mu^{*}$ с константами $\sigma^{*}$

\begin{tabular}{|c|c|c|c|c|}
\hline Заместитель & $\frac{\mu_{\mathrm{R}}}{1,95}\left[{ }^{8}\right]$ & $\frac{\mu^{\prime}}{1,85}\left[{ }^{8}\right]$ & $\begin{array}{c}\mu^{*} \text { по } \\
\text { формуле (1) }\end{array}$ & $\sigma^{*}\left[{ }^{5}\right]$ \\
\hline $\begin{array}{l}\mathrm{CH}_{3} \\
\mathrm{C}_{2} \mathrm{H}_{5} \\
\mathrm{C}_{3} \mathrm{H}_{7} \\
\mathrm{C}_{4} \mathrm{H}_{9} \\
\mathrm{C}_{5} \mathrm{H}_{11} \\
i-\mathrm{C}_{3} \mathrm{H}_{7} \\
\mathrm{CH}\left(\mathrm{C}_{2} \mathrm{H}_{5}\right)_{2} \\
i-\mathrm{C}_{4} \mathrm{H}_{9} \\
i-\mathrm{C}_{5} \mathrm{H}_{11} \\
\mathrm{C}\left(\mathrm{CH}_{3}\right)_{3} \\
\mathrm{C}\left(\mathrm{C}_{2} \mathrm{H}_{5}\right)\left(\mathrm{CH}_{3}\right)_{2} \\
\left(\mathrm{CH}_{2}\right)_{\infty} \mathrm{CH}_{3}\end{array}$ & $\begin{array}{l}0 \\
-0,100 \pm 0,014 \\
-0,148 \pm 0,016 \\
-0,173 \pm 0,018 \\
-0,180 \\
-0,187 \\
-0,194 \pm 0,019 \\
-0,190 \\
-0,264 \pm 0,008 \\
-0,310 \\
-\end{array}$ & $\begin{array}{c}0 \\
-0,1 \\
-0,153 \\
-0,199 \\
-0,196 \\
-0,207 \\
-0,270 \\
-\end{array}$ & $\begin{array}{c}0 \\
-0,1 \\
-0,15 \\
-0,175 \\
-0,188 \\
-0,20 \\
-0,30 \\
-0,20 \\
-0,20 \\
-0,30 \\
-0,35 \\
-0,20\end{array}$ & $\begin{array}{l}0 \\
-0,1 \\
-0,115 \\
-0,13 \\
-0,19 \\
-0,225 \\
-0,125 \\
-0,30 \\
-\end{array}$ \\
\hline
\end{tabular}

Из табл. 1 следует, что значения констант $\sigma^{*}$ Тафта при удлинении углеродной цепи изменяются меньше значений констант $\mu$.

Предметом особого внимания является водородный заместитель. Авторы работы $\left[{ }^{8}\right]$ утверждают, что значение $\mu^{\prime}$ для водорода варьируется в широких пределах. Однако из табл. 6 этой же работы видно, что кроме двух предельных случаев - альдегидов-кетонов и аминов, значения $\mu^{\prime}$ для водорода довольно близки. Альдегидам-кетонам соответствует значение $\frac{\mu^{\prime}}{1,85}=0,22$ и аминам $\frac{\mu^{\prime}}{1,85}=0,96$. Для шести остальных серий соединений получается $\frac{\mu^{\prime}}{1,85}=0,51 \pm 0,06$. Относительная ошибка этой величины не превышает ошибки констант алкильных заместителей. Величина 0,51 близка к тафтовскому значению $\sigma^{*}=0,49$.

Результат для серии аминов можно считать сомнительным, так как точные значения их адиабатических потенциалов ионизации не известны. Серии альдегидов-кетонов отличаются от других рассмотренных серий тем, что у них водород связан с центром ионизации не прямо, а через углеродный атом карбонильной группы.

Как предполагалось в $\left[{ }^{1,8}\right]$, величина изменения ПИ, вызванного введением заместителя, является подходящей относительной мерой

таблица 2

\begin{tabular}{|c|c|}
\hline Серия & $\begin{array}{c}\mu_{\text {H }}^{*} \\
\text { по формуле (2) }\end{array}$ \\
\hline $\begin{array}{l}\mathrm{H}_{2} \mathrm{C}=\mathrm{CH}-\mathrm{X}^{* * *} \\
\mathrm{HC} \equiv \mathrm{C}-\mathrm{X} \\
\mathrm{Cl}-\mathrm{X} \\
\mathrm{Br}-\mathrm{X} \\
\mathrm{J}-\mathrm{X} \\
\mathrm{HO}-\mathrm{X} \\
\mathrm{HS}-\mathrm{X} \\
\mathrm{CH} \mathrm{H}_{3} \mathrm{~S}-\mathrm{X} \\
\mathrm{CN}-\mathrm{X}\end{array}$ & $\begin{array}{l}0,52 \pm 0,07 \\
0,58 \pm 0,07 \\
0,49 \pm 0,07 \\
0,45 \pm 0,04 \\
0,40 \pm 0,04 \\
0,47 \pm 0,09 \\
0,68 \pm 0,09 \\
0,56 \pm 0,06 \\
0,45 \pm 0,04\end{array}$ \\
\hline $\begin{array}{l}\text { Среднее } \\
\text { взвешенное }\end{array}$ & $0,48 \pm 0,02$ \\
\hline
\end{tabular}
оценки его индукционного влияния. Это позволяет вычислять значение $\mu_{\mathrm{H}}^{*}$ несколько иначе, а именно по формуле (2), заменяя величину $I_{\mathrm{RY}}$ величиной ПИ соединения с водородным заместителем $I_{\text {ну. }}$ Этот способ дает возможность рассматривать больше серий, чем в $\left.{ }^{8}\right]$.

В настоящей работе расчет $\mu_{\mathrm{H}}^{*}$ был проведен для девяти серий (см. табл. 2). Исходные данные ПИ взяты из $\left[{ }^{12}\right]$, и возможные отклонения рассчитаны по погрешности исходных ПИ. Вели- 
чины $\mu_{H}^{*}$ по табличным данным можно считать приблизительно постоянными и среднее взвешенное для девяти серий равно $0,48 \pm 0,02$. Так как по результатам работы $\left[{ }^{8}\right]$ получено значение $\mu_{\mathrm{H}}^{*}=0,51$, то кажется целесообразным применение совпадающего с константой $\sigma^{*}$ Тафта значения $\mu^{*}=0,49$ для водородного заместителя.

В системе констант $\mu^{*}$ водородного и алкильных заместителей общий закон затухания, как это обнаружено в случае констант $\sigma^{*}$ Тафта $\left[{ }^{13}\right]$, не действует. Фактор затухания при переходе от водорода к метильному заместителю равен 0,2 (см. табл. 1 в $\left[{ }^{7}\right]$ ), а для алкильных заместителей $-0,5\left[{ }^{7}\right]$. Если в зависимости $\sigma_{\left(\mathrm{xcH}_{2}\right)}^{*}=-0,100+0,204 \sigma_{(\mathrm{X})}^{*}\left[{ }^{13}\right]$ вместо $z=0,204$ взять $z=0,5$, то для алкильных заместителей получим результаты, совпадающие с уравнением (1).

\section{Применение констант $\mu^{*}$ для алифатических соединений}

В настоящей работе коррелируются ПИ с константами $\mu^{*}$ и $\sigma^{*}$ в случае алкил- и водородозамещенных 1-алкенов, 1-алкинов и алифатических соединений с гетероатомом по уравнениям (7) и (7A):

$$
\begin{aligned}
& I=I_{0}+\mathrm{Q}^{*} \mu^{*}, \\
& I=I_{0}+\mathrm{Q}^{*} \sigma^{*},
\end{aligned}
$$

где $I_{0}-$ ПИ соединения с метильным заместителем, $\rho^{*}-$ постоянная чувствительности индукционного эффекта данной серии.

Для двухзамещенных соединений, например для эфиров, тиоэфиров и кетонов, вместо индукционных констант взята сумма констант обоих заместителей $\Sigma \mu^{*}$ или $\Sigma \sigma^{*}$. Параметры уравнений $I_{0}$ и $\rho^{*}$ определены методом наименьших квадратов и представлены в табл. 3 . Там же приведены значения коэффициента корреляции $r$ и стандартного отклонения $s$. Использованы только данные ПИ фотоионизационного метода, полученные из обзорной статьи $\left[{ }^{2}\right]$ и из работ $\left[{ }^{6,12,14-16}\right]$. Величины $\sigma^{*}$ взяты 13 [5], но, к сожалению, они не определены для всех алкильных заместителей. Как видно из табл. 3, для всех 11 серий качество корреляции с кснстантами $\mu^{*}$ по уравнению (7) намного лучше, чем с константами $\sigma^{*}$ по уравнению (7A). Остановимся подробнее на корреляции с велнчинами $\mu^{*}$. Аналогичные корреляции для семи серий без учета водородного заместителя представлены в табл. 3 работы $\left[{ }^{7}\right]$. В результате включения водородного заместителя стандартное отклонение увеличивается, но все-таки не превышает для семи серий 0,03 эв. Значительно расширяется диапазон изменений, относительная ошибка остаетгя неизменной и коэффициент корреляции улучшается, кроме серий альдегидов и кетонов (для девяти серий $r>0,99$ ).

Ухудшение качества корреляции у альдегидов и кетонов в результате включения водорода естественно, поскольку для этих серий величина $\mu^{*}=0,49$ слишком груба, как было показано выше.

В случае присоединения к гетероатому более одного алкильного заместителя наблюдается «эффект насыщения» - влияние второго заместителя меньше первого. Так, для серий $\mathrm{R}-\mathrm{OH}$ и $\mathrm{R}^{1}-\mathrm{O}-\mathrm{R}^{2}$ соответствующие величины чувствительности $\rho^{*}$ равны 3,60 и 1,94 , а в случае серий $\mathrm{R}-\mathrm{SH}$ и $\mathrm{R}^{1}-\mathrm{S}-\mathrm{R}^{2}-1,99$ и 1,40 .

\section{Применение констант $\mu^{*}$ для циклопентенов и циклогексенов}

В работе $\left[{ }^{17}\right]$ представлены ПИ большого количества алкилзамещенных циклопентенов и -гексенов, определенные методом фотоионизации, и проанализирована их зависимость от структурных факторов, 


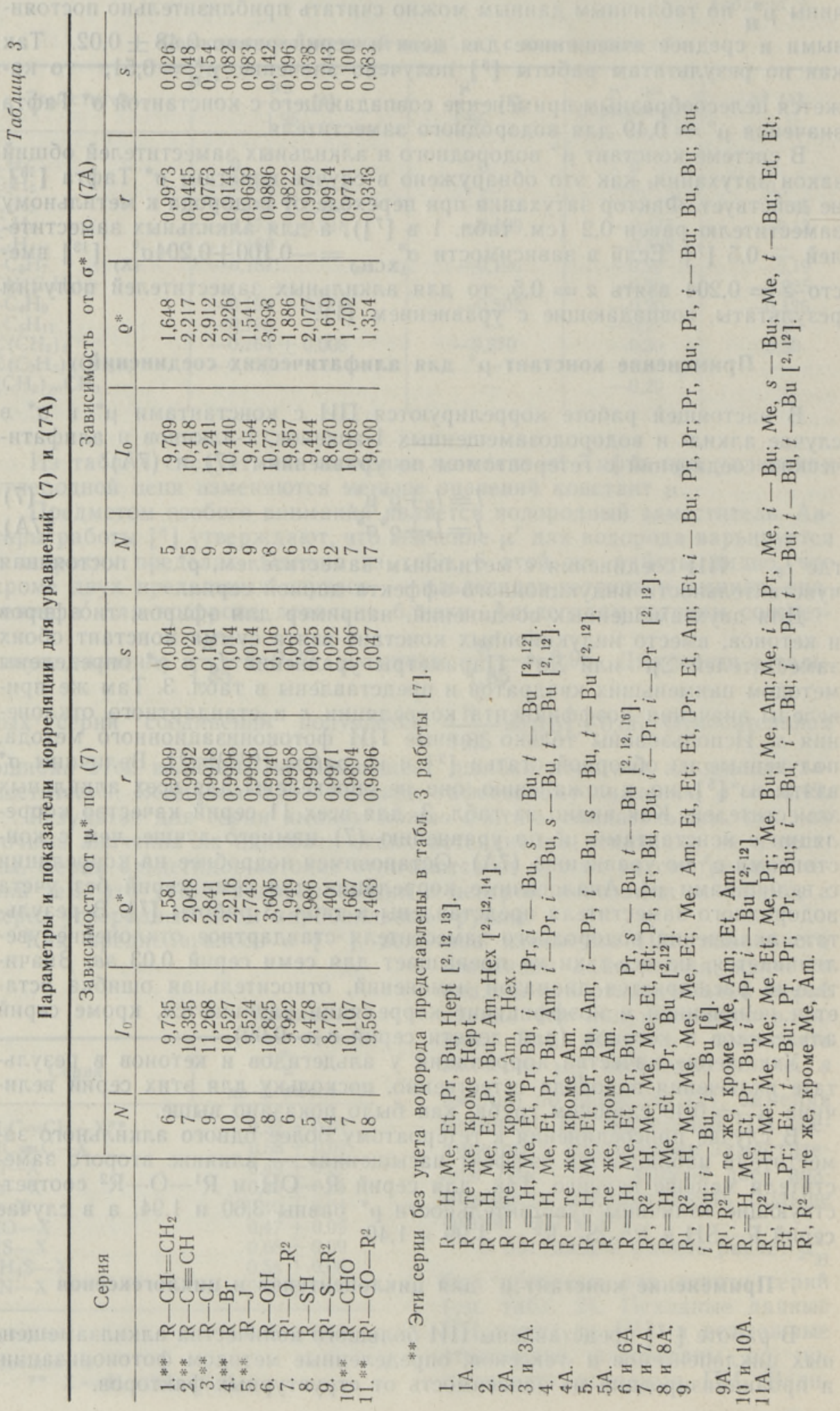




\begin{tabular}{|c|c|c|c|}
\hline $\begin{array}{c}\text { Цикличе } \\
{\left[\left(\mathrm{CH}_{2}\right)_{n}\right]} \\
\mathrm{HC} \stackrel{\mathrm{CH}}{=},\end{array}$ & $\begin{array}{l}\text { алкены } \\
\quad n \geqslant 3\end{array}$ & $\begin{array}{r}\text { Алифа } \\
\mathrm{H}\left(\mathrm{CH}_{2}\right)_{l} \mathrm{HC}=\end{array}$ & $\begin{array}{l}\text { кие алкены } \\
\left.\mathrm{CH}_{2}\right)_{m} \mathrm{H}, l+m=n\end{array}$ \\
\hline Соединение & $I, \quad 98$ & Соединение & $I, \quad ә 8$ \\
\hline $\begin{array}{l}\text { Циклопентен } \\
\text { 3-Метил-1-цикло- } \\
\text { пентен } \\
\text { Циклогексен } \\
\text { 1-Этил-1-цикло- } \\
\text { гексен } \\
\text { Циклооктен } \\
\text { Циклодецен }\end{array}$ & $\begin{array}{l}9,02 \pm 0,01\left[{ }^{17}\right] \\
8,95 \pm 0,01\left[{ }^{17}\right] \\
8,945 \pm 0,01 \\
\left.{ }^{12}\right] \\
8,48 \pm 0,01\left[{ }^{17}\right] \\
8,82 \\
8,80\end{array}$ & $\begin{array}{l}\text { цис-2-Пентен } \\
\text { цис-4-Метил- } \\
-2-\text {-пентен } \\
\text { цис-3-Гексен } \\
\text { 3-Этил-3-гексен } \\
\text { цис-4-Октен } \\
\text { цис-5-Децен }\end{array}$ & $\begin{array}{l}9,036 \pm 0,005\left[{ }^{18}\right] \\
8,976 \pm 0,005\left[{ }^{18}\right] \\
8,954 \pm 0,005\left[{ }^{18}\right] \\
8,480 \pm 0,004\left[{ }^{18}\right] \\
8,836 \pm 0,006\left[{ }^{13}\right] \\
8,773 \pm 0,006\left[{ }^{13}\right]\end{array}$ \\
\hline
\end{tabular}

При сопоставлении ПИ соответствующих алифатических и циклических алкенов видно, что данные практически совпадают (табл. 4).

По-видимому, у алифатических и циклических алкенов, начиная с циклопентена (малонапряженные циклы), действуют общие закономерности в изменении ПИ.

Между ПИ алкилзамещенных этиленов $\mathrm{X}_{1} \mathrm{X}_{2} \mathrm{C}=\mathrm{CX}_{3} \mathrm{X}_{4}(\mathrm{X}-\mathrm{H}$ или алкилзаместитель) и суммой индукционных констант всех четырех заместителей $\Sigma \sigma_{I}$ наблюдается линейная зависимость, как установлено в работе $\left[{ }^{9}\right]$. В принципе эта зависимость не может быть точной, так как согласно [20,21] при замещении в этилене водорода метильными группами аддитивность соблюдается очень грубо. Последовательное замещение водорода метильными группами вызывает не равное падение ПИ, а явление «насыщения». При введении первой, второй, третьей и четвертой метильных групп в этилен падение ПИ равно соответственно 0,78 ; 0,$60 ; 0,45$ и 0,38 эв. Так как разница в изменении двух последних замещений сравнительно мала, то можно довольно точно скоррелировать ПИ соединений типа $\mathrm{R}_{1} \mathrm{HC}=\mathrm{CHR}_{2}(\mathrm{I}), \mathrm{R}_{1} \mathrm{R}_{3} \mathrm{C}=\mathrm{CHR}_{2}$ (II) и $\mathrm{R}_{1} \mathrm{R}_{3} \mathrm{C}=$ $=\mathrm{CR}_{3} \mathrm{R}_{4}(\mathrm{III})$ (здесь $\mathrm{R}$ - алкильная группа) с суммой индукционных констант всех заместителей.

В настоящей работе циклопентены и -гексены рассматриваются как алкены с открытой углеродной цепью, полученной при пересечении цикла на максимальном расстоянии от двойной связи, т. е. между четвертым и пятым положениями. Таким образом, все монозамещенные циклические алкены можно принять в качестве замещенных этиленов (I) и (II) типа. Сумма индукционных констант заместителей найдена по всем четырем заместителям с применением для водорода значения $\mu^{*}=0,49 ; \mu^{*}$ для всех алкильных заместителей вычислялось по формуле (1). Циклогексиловый заместитель рассматривался как $\mathrm{CH}\left(\mathrm{C}_{2} \mathrm{H}_{5}\right)\left(\mathrm{C}_{3} \mathrm{H}_{7}\right)$, и по формуле (1) было получено $\mu^{*}=-0,325$. Вычисление по формуле (7) из ПИ бромистого циклогексана 9,85 эв $\left[{ }^{2}\right]$ дает результат $\mu^{*}=-0,31$ (см. табл. 3, серия 4). Эти две оценки $\mu^{*}$ хорошо согласуются между собой, но они несколько отрицательнее значения $\sigma^{*}=-0,26\left[{ }^{22}\right]$, полученного из данных щелочного и кислотного гидролиза этилового эфира циклокарбоновой кислоты.

Зависимость ПИ циклопентенов и -гексенов от величин $\Sigma \mu^{*}$ выражается следующими уравнениями:

$$
\begin{aligned}
& I=8,274+0,827 \Sigma \mu^{*}, \\
& N=18, \quad r=0,997, \quad s=0,016 \quad \text { эв; }
\end{aligned}
$$


Таблица 5

Экспериментальные и вычисленные Пи циклопентенов

\begin{tabular}{|c|c|c|c|c|}
\hline \multirow[b]{2}{*}{ Соединения } & \multirow[b]{2}{*}{$\Sigma \mu^{*}$} & \multirow{2}{*}{$\begin{array}{c}I, \quad 9 B \\
{\left[{ }^{17}\right]}\end{array}$} & \multicolumn{2}{|c|}{ I } \\
\hline & & & по $(8)$ & nо $(10)$ \\
\hline Циклопентен & 0,88 & 9,02 & 9,00 & 9,01 \\
\hline 1-Метил-1-цнклопентен & 0,39 & 8,60 & 8,60 & 8,61 \\
\hline 1-Этил-1-циклопентен & 0,29 & 8,53 & 8,51 & 8,53 \\
\hline 1-н-Пропил-1-циклопентен & 0,24 & 8,48 & 8,47 & 8,49 \\
\hline 1-н-Бутил-1-циклопентен & 0,215 & 8,45 & 8,45 & 8,47 \\
\hline 1-н-Пентил-1-циклопентен & 0,202 & 8,45 & 8,44 & 8,46 \\
\hline 1 -н-Гексил-1-циклопентен & 0,196 & 8,43 & 8,44 & 8,45 \\
\hline 1-н-Гептил-1-циклопентен & 0,193 & 8,41 & 8,43 & 8,45 \\
\hline 1-Изобутил-1-циклопентен & 0,19 & 8,44 & 8,43 & 8,45 \\
\hline 1-Изопентил-1-циклопентен & 0,19 & 8,44 & 8,43 & 8,45 \\
\hline 3-Метил-1-циклопентен & 0,78 & 8,95 & 8,92 & 8,93 \\
\hline 3-Этил-1-циклопентен & 0,73 & 8,88 & 8,88 & 8,89 \\
\hline 3-н-Пропил-1-циклопентен & 0,703 & 8,84 & 8,86 & 8,87 \\
\hline 3-н-Бутил-1-циклопентен & 0,692 & 8,83 & 8,85 & 8,86 \\
\hline 3-н-Пентил-1-циклопентен & 0,686 & 8,84 & 8,84 & 8,85 \\
\hline 3-н-Гексил-1-циклопентен & 0,683 & 8,84 & 8,84 & 8,85 \\
\hline 3-Изопропил-1-циклопентен & 0,68 & 8,81 & 8,84 & 8,85 \\
\hline 3-Изопентил-1-циклопентен & 0,68 & 8,83 & 8,84 & 8,85 \\
\hline
\end{tabular}

Экспериментальные и вычисленные Пи циклогексенов

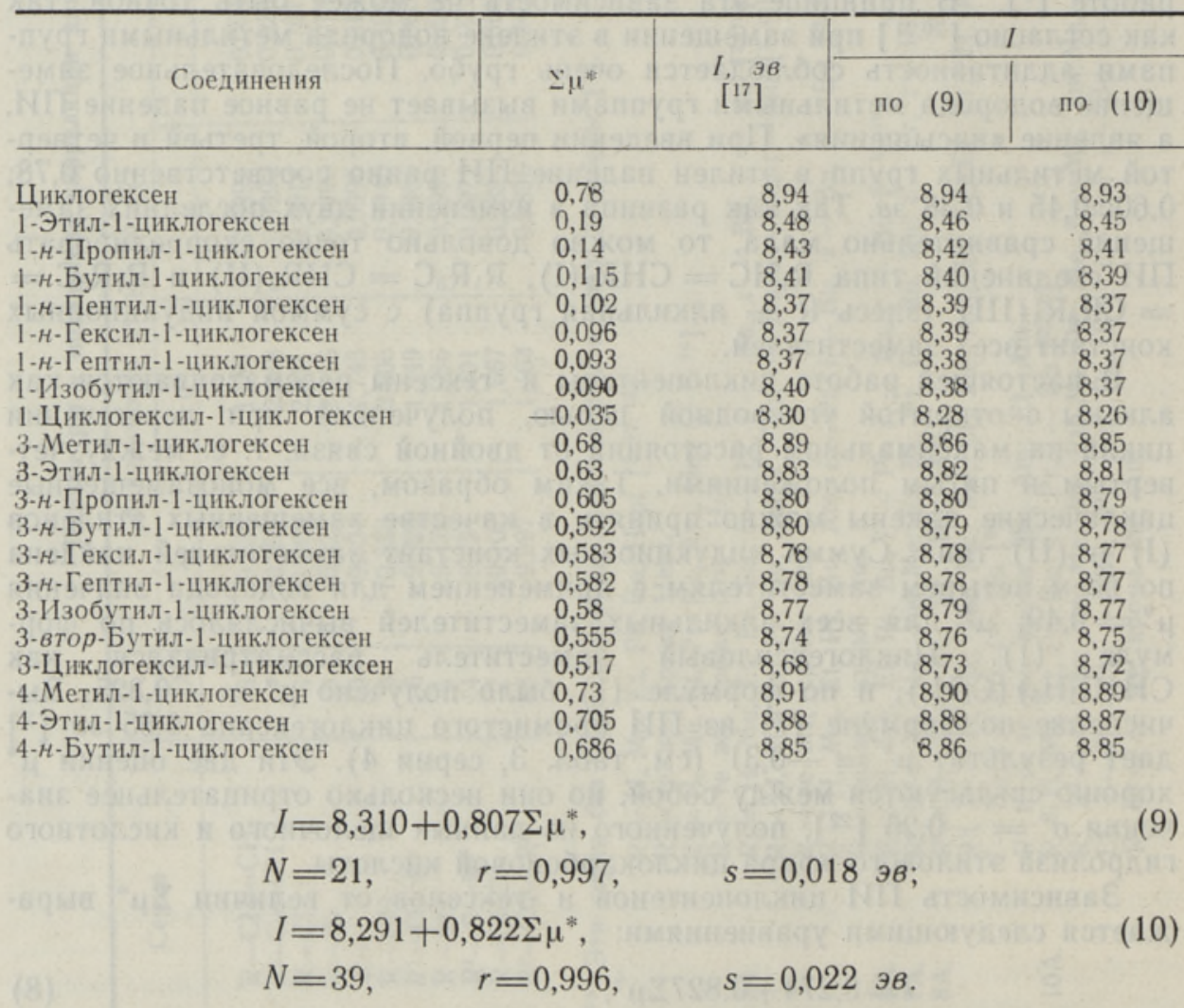

Уравнение (8) описывает циклопентены, (9) - циклогексены 
(10) - общее для них. Отрезок ординаты в этих уравнениях соответствует ПИ 2,3-диметил-2-бутена $\left(\mathrm{CH}_{3}\right)_{3} \mathrm{C}=\mathrm{C}\left(\mathrm{CH}_{3}\right)_{3}$, экспериментальное значение которого $8,30 \pm 0,02\left[{ }^{23}\right]$. Сопоставление экспериментальных данных ПИ циклопентенов и -гексенов $\left[{ }^{17}\right]$ со значениями, вычисленными по уравнениям $(8)-(10)$, показывает их хорошее согласие между собой (табл. 5 и 6). Уравнение (8) дает отклонение выше 0,02 эв только у двух циклопентенов: 3-метил-1-циклопентена $(-0,03$ эв) и 3 изопропил-1-циклопентена $(+0,03$ эв), а уравнение $(9)$ только у двух циклогексенов: 3-циклогексил-1-циклогексена $(+0,05$ эв) и 3 -метил-1циклогексена $(-0,03$ эв).

Для высших циклоалкенов по формуле (10) имеем следующие значения ПИ: циклогептен 8,89 эв, циклооктен 8,85 эв и циклодецен 8,81 эв. Соответствующие экспериментальные результаты, полученные методом фотоэлектронной спектроскопии, хорошо согласуются с ними: 8,$87 ; 8,82$ и 8,80 эв $\left[{ }^{19}\right]$.

Интересно отметить, что формула (10) с хорошей точностью позволяет вычислять ПИ 2,3-диметил-2-бутена, 3-метил-2-бутена и 2-бутена, хотя ПИ названных соединений не были использованы при вычислении параметров уравнений. Так, вычисленные и экспериментальные значения ПИ этих соединений равны соответственно: $8,29 \quad\left(8,30 \pm 0,01\left[{ }^{23}\right]\right)$, $8,69\left(8,67 \pm 0,01\left[{ }^{12}\right]\right)$ и $9,10\left(9,13 \pm 0,01\left[{ }^{12}\right]\right)$. Уравнение $(10)$ не применимо по указанным выше причинам к 1-пропену и этилену. Для этих соединений отклонения от экспериментальных данных достигают 0,23 и 0,61 эв соответственно.

\section{Выводы}

1. Константы $\mu^{*}$, вычисленные на основе закономерности затухания и аддитивности индукционного влияния, хорошо согласуются с константами $\mu_{R}$, полученными по снижению потенциала ионизации, вызванному алкильным заместителем.

2. Система констант $\mu^{*}$ дополнена величиной $\mu^{*}=0,49$ для водорода, полученной по изменению потенциала ионизации.

3. Значения $\mu^{*}$ для циклогексилового радикала, полученные двумя различными методами, равны $-0,325$ и $-0,31$.

4. Зависимость потенциалов ионизации алифатических соединений с водородным и алкильным заместителями от констант $\sigma^{*}$ Тафта близка к линейной лишь приблизительно, в случае же констант $\mu^{*}$ точность ее заметно улучшается.

5. Величина влияния алкильного заместителя или углеродного цикла (в случае циклоалкенов) на потенциал ионизации в основном определяется числом углеродных атомов и их расстоянием по С-С-связям от хромофорной группы.

\section{ЛИТЕРАТУРА}

1. В илес о в Ф. И., Докт. дис., Л., 1966.

2. Вилесов Ф. И., В сб.: Успехи фотоники, Вып. 1., Л., 1969, с. 5.

3. Ингольд К. К., Механизм реакций и строение органических соединений, гл. 2., M. 1959.

4. Branch G. E., C a I vin M., The Theory of Organic Chemistry, New York, 1941, p. 217.

5. Т а ф т Р., В кн.: Пространственные эффекты в органической химии, гл. ХIII, M. 1960 .

6. Акопя н М. Е., ЖФХ, 44, 2918 (1970).

7. Пылдоя П., Изв. АН ЭССР, Хим. Геол., 1976 (в печати).

8. Cocksey B. J., E 1 a nd J. H., D a n b y C. J., J. Chem. Soc. (B), 790 (1970) 
9. Levitt L. S., Levitt B. W., Párkányi C., Tetrahedron, 28, 3369 (1972).

10. Levitt L. S., Levitt B. W., J. Org. Chem., 37, 332 (1972).

11. Levitt L. S., Levitt B. W., Tetrahedron, 29, 941 (1973).

12. Wat a n abe K., Nakajama T., Mottl J., J. Quant. Spectrosc. Radiat. Transfer, 2, 369 (1962).

13. П альм В. А., Успехи химии, 30, 1069 (1961)

14. Р ан С., Мартинсон Э., Мюрисепп М., Изв. АН ЭССР. Хим. Геол. 23,352 (1974).

15. Виллем Я.Я., Пиквер Р. И., С акс О. В., Тезисы 3-й Всесоюзн. конф. по спектроскопии вакуумного ультрафиолета и взаимодействия излучения с вешеством, Харьков, 1972 , с. 65.

16. Is a a cs L. D., Price W. C., Ridley R. G., The threshold of space, London, Pergamon Press, 1957, p. 143.

17. РангС., Пылдоя П., Т алвари А., Изв. АН ЭССР, Хим. Геол., 23, 354 (1974).

18. Masclet W., Grosjean D., Mouvier D., D ubois J., J. of Electron Spectroscopy and Related Phenomena, 2, 225 (1973)

19. B is hof P. Heilbronner E., Helv. Chim. Acta, 53, 1677 (1970).

20. Price W. C., Brals ford R., Har is P. V., Ridley R. G., Spectrochim. Acta, $14,45(1959)$.

21. В и лесов Ф. И., Успехи физических наук, 81, 669 (1963).

22. Wilcox C. F., J. Org. Chem., 27, 2210 (1962).

23. Pric e W. C., Chem. Rev. 41, 257 (1947).

\section{Ннститут физики \\ Академии наук Эстонской ССР \\ Поступила в редакцию $14 / \mathrm{I} \quad 1975$}

\section{P. POLDOJA}

\section{VESINIKU JA ALKUULASENDAJA MÕUST IONISATSIOONIPOTENTSIAALILE}

Uuriti vesiniku ja alküülasendaja mōju alifaatsete ühendite ja tsükloalkeenide ionisatsioonipotentsiaalile. Ingoldi, Branchi ja Calvini induktsioonefekti teooria põhjal arvutatud $\mu^{*}$ konstandid on heas kooskõlas ionisatsioonipotentsiaali muutuse alusel saadud $\mu_{\mathrm{R}}$ konstantidega. Alifaatsete ühendite 11 seeria puhul näidatakse, et ionisatsioonipotentsiaalid on Tafti $\sigma^{*}$ induktsioonkonstantidest ligikaudses ja $\mu^{*}$ konstantidest heas lineaarses sōltuvuses. Nii alküülasendaja kui ka tsükloalkeenide süsivesinikutsükli mõju suuruse ionisatsioonipotentsiaalile määrab põhiliselt süsinikuaatomite arv ja kaugus kromofoorsest grupist C - C-sidemeid pidi, mis on kooskõlas induktsioonefekti teooriaga.

\section{P. POLDOJA}

\section{ON THE EFFECT OF HYDROGEN AND ALKYL SUBSTITUENT ON THE IONIZATION POTENTIAL}

The effect of hydrogen and alkyl substituents on the ionization potential of aliphatic compounds and cycloalkenes is studied. $\mu^{*}$ constants calculated on the basis of the Ingold, Branch and Calvin induction effect theory are found in good agreement with $\mu_{\mathrm{R}}$ constants found from the variation of ionization potentials. Approximately linear dependence of ionization potentials on Taft's $\sigma^{*}$ constants and good linear dependence on $\mu^{*}$ constants are demonstrated in case of 11 aliphatic series. The effect of both alkyl substituent and carbon cycle on the ionization potentials depends mainly on the number of carbon atoms and on the distance from the chromophoric group via $\mathrm{C}-\mathrm{C}$ bonds as predicted by the induction effect theory. 